\title{
The Associations between Visual Attention and Facial Expression Identification in Patients with Schizophrenia
}

\author{
I-Mei Lin ${ }^{1}$, Sheng-Yu Fan ${ }^{2,3} \bowtie$, Tiao-Lai Huang ${ }^{4}$, Wan-Ting Wu', and Shi-Ming Li ${ }^{5}$ \\ ${ }^{1}$ Department of Psychology, Kaohsiung Medical University, Kaohusiung, Taiwan \\ ${ }^{2}$ Department of Human Development, Tzu Chi University, Hualien, Taiwan \\ ${ }^{3}$ Institute of Gerontology, National Cheng Kung University, Tainan, Taiwan \\ ${ }^{4}$ Department of Psychiatry, Kaohsiung Chang Gung Memorial Hospital and Chang Gung University College of Medicine, Kaohusiung, Taiwan \\ ${ }^{5}$ Department of Clinical Psychology, Shin-Ann Hospital, Yunlin, Taiwan
}

\begin{abstract}
Objective Visual search is an important attention process that precedes the information processing. Visual search also mediates the relationship between cognition function (attention) and social cognition (such as facial expression identification). However, the association between visual attention and social cognition in patients with schizophrenia remains unknown. The purposes of this study were to examine the differences in visual search performance and facial expression identification between patients with schizophrenia and normal controls, and to explore the relationship between visual search performance and facial expression identification in patients with schizophrenia.
\end{abstract}

Methods Fourteen patients with schizophrenia (mean age $=46.36 \pm 6.74$ ) and 15 normal controls (mean age $=40.87 \pm 9.33$ ) participated this study. The visual search task, including feature search and conjunction search, and Japanese and Caucasian Facial Expression of Emotion were administered.

Results Patients with schizophrenia had worse visual search performance both in feature search and conjunction search than normal controls, as well as had worse facial expression identification, especially in surprised and sadness. In addition, there were negative associations between visual search performance and facial expression identification in patients with schizophrenia, especially in surprised and sadness. However, this phenomenon was not showed in normal controls.

Conclusion Patients with schizophrenia who had visual search deficits had the impairment on facial expression identification. Increasing ability of visual search and facial expression identification may improve their social function and interpersonal relationship.

Psychiatry Investig 2013;10:393-398

Key Words Schizophrenia, Visual search, Facial expression identification.

\section{INTRODUCTION}

Previous studies have extensively verified cognition function impairment in patients with schizophrenia. ${ }^{1-3}$ Cognition function impairment was found on not only executive function, verbal fluency, process speed, visual attention, but also social cognition. ${ }^{4-7}$ According to the information-processing theory $^{8}$ and the feature-integration theory, ${ }^{9}$ visual attention is the

Received: October 14, 2012 Revised: November 25, 2012

Accepted: December 14, 2012 Available online: December 16, 2013

$\triangle$ Correspondence: Sheng-Yu Fan, PhD

Institute of Gerontology, National Cheng Kung University, No. 1, University Road, Tainan City 701, Taiwan

Tel: +886-6-2353535 ext. 5737, Fax: $+886-6-3028715$

E-mail: dibe1011@yahoo.com.tw

(c) This is an Open Access article distributed under the terms of the Creative Commons Attribution Non-Commercial License (http://creativecommons.org/licenses/by$\mathrm{nc} / 3.0$ ) which permits unrestricted non-commercial use, distribution, and reproduction in any medium, provided the original work is properly cited. first step of information process, which means our gaze rapidly towards objects in our visual environment field, through bottom-up and top-down process integrate the visual information. ${ }^{10}$ It includes two core components: 1 ) feature search: the functioning of the preattentive and parallel process, participants are required to search a target item among a number of non-target items (e.g., color); and 2) conjunction search: the functioning of the focal attention and serial process, participants are required to search a target item among a number of non-target items with two type of distractors (e.g., color and display size)..$^{11-13}$ The deficits of visual attention indicated as the endure elements across the lifetime of schizophrenia. ${ }^{9}$

Visual search deficit is considered a core symptom of visual attention impairment that interrupts cognition process and social cognition in patients with schizophrenia. ${ }^{14}$ Tanaka et al. ${ }^{3}$ explored the detection and recognition of visual search per- 
formance between patients with schizophrenia and normal controls. The results showed that there was no group difference in feature search; however, conjunction search deficit was found in patients with schizophrenia. ${ }^{3}$ Less efficient on visual search task was a deficit in perceptual organization. ${ }^{15}$ However, Tanaka et al. ${ }^{3}$ did not examine the relationship between the visual search performance and social cognition.

Social cognition can be understood as "the mental operations underlying social interactions, which include the human ability to perceive the intentions and dispositions of others"16 Facial expression identification is one of social cognition ability which includes decoding, encoding, and subjective reactions of facial expressions of emotions. ${ }^{17}$ Kerr and Neale ${ }^{18}$ found that patients with schizophrenia had worse emotion recognition than normal controls. Addington and Addington ${ }^{19}$ found that patients with schizophrenia performed poorly on facial affect recognition and facial recognition than bipolar patients and normal controls.

Visual attention is an early-stage of visual processing which through bottom-up approach related to emotion recognition. ${ }^{20}$ Kalkstein et al. ${ }^{21}$ demonstrated the facial expression and recognition deficits in patients with schizophrenia. Deficits emotion identification was associated with impaired the magnocellular-basis visual sensory processing. ${ }^{20}$ The visual attention deficits may associate with facial affect recognition deficits. ${ }^{19}$

What is the role of visual attention in facial expression identification? A visual attention deficit may influence information processing and cause cognition deficit, and may even result in poor social cognition..$^{22}$ Social cognition is mediated by cognition function (e.g., attention, memory, executive function) which influences the outcome of social function (e.g., social relationship and social skill) ${ }^{23}$ However, there are few studies that have linked visual search performance and facial expression identification in patients with schizophrenia.

\section{Aims of the study}

The aims of this study were: 1) to examine the differences in visual search performance between patients with schizophre- nia and normal controls; 2) to examine the differences in facial expression identification between patients with schizophrenia and normal controls; and 3) to explore the relationship between cognition (visual search performance) and social cognition (facial expression identification).

\section{METHODS}

\section{Participants}

Fourteen patients with schizophrenia (mean age $=46.36 \pm$ 6.74; 8 male and 6 female) and fifteen normal controls (mean age $=40.87 \pm 9.33 ; 4$ male and 11 female) participated in this study. Patients with schizophrenia were diagnosed by psychiatrists based on the criteria of Diagnostic and Statistical Manual of Mental Disorders-IV-TR (DSM-IV-TR). ${ }^{24}$ Fifteen patients with schizophrenia were recruited from the Shin-Ann Hospital, one were being excluded due to interrupt by psychiatric symptoms and cannot follow the experimental procedure. Normal controls were health population who were recruited form community.

All participants were excluded color blindness, substance abuse, others psychiatry disorders, and severe clinical illness. The age, gender, years of education, the duration of schizophrenia diagnosis from onset to the experiment, and the length of the current hospitalization were showed in Table 1. There were no significant differences in age, gender, and years of education between patients with schizophrenia and normal controls $\left(\mathrm{t}=-1.81, \mathrm{p}>0.05 ; \chi^{2}=2.77, \mathrm{p}>0.05 ; \mathrm{t}=1.54, \mathrm{p}>0.05\right.$ respectively). Institutional Review Board (IRB) approval was obtained from the ethics committee of Shin-Ann Hospital and informed consent were obtained from all participants before study.

\section{Measures}

\section{The visual search task}

The visual search task (VST) was used to measure cognition function, including feature search task and conjunction search

Table 1. Demographic information of patients with schizophrenia and normal controls

\begin{tabular}{|c|c|c|c|}
\hline & $\begin{array}{l}\text { Patients with schizophrenia } \\
\qquad(\mathrm{N}=14)\end{array}$ & $\begin{array}{l}\text { Normal controls } \\
\qquad(\mathrm{N}=15)\end{array}$ & $t / x^{2}$ \\
\hline Age (years) & $46.36(\mathrm{SD}=6.74)$ & $40.87(\mathrm{SD}=9.33)$ & $\mathrm{t}=-1.81$ \\
\hline Gender & & & $\chi^{2}=2.77$ \\
\hline Male & $8(57.14 \%)$ & $4(26.67 \%)$ & \\
\hline Female & $6(42.86 \%)$ & $11(73.33 \%)$ & \\
\hline Years of education (years) & $9.43 \pm 2.59$ & $11.07 \pm 3.11$ & $\mathrm{t}=1.54$ \\
\hline Duration of disorder (range $=156-360$ months) & $235.20 \pm 70.93$ & N/A & \\
\hline Length of the current hospitalization (range=1-20 months) & $10.00 \pm 4.49$ & N/A & \\
\hline
\end{tabular}


task. The target stimulus in both tasks was a red square; the distractors were red circles in the feature search task and red circles and green squares in the conjunction search task (Figure 1). The experimental material and procedure were followed by Tanaka et $\mathrm{al}^{3}{ }^{3}$ In each task, a set of 4, 16, or 25 items was presented, and each task included one practice block of 10 trials and three blocks of 30 trials each.

Total was 100 stimuli for the feature search task and 100 stimuli for conjunction search task. The red square of target was randomly displayed in half of each trial. Reaction times (RTs) and the numbers of correct response (accuracy rate) were recorded.

\section{Japanese and Caucasian Facial Expression of Emotion} Japanese and Caucasian Facial Expression of Emotion (JACFEE) was used to measure the participants' ability to process the facial expression identification. ${ }^{25,26}$ There are six different emotional expressions, anger, fear, happiness, surprised, disgusted, and sadness; and each emotion has four pictures. Twenty-four different faces were randomly presented and the accuracy rates of each emotion expression were recorded.

\section{Experimental procedure}

Participants finished VAS first and then JACFEE. This study used a 14-inch laptop with Superlab software (Cedurs Corporation, San Pedro, CA) for displaying the visual search stimuli. Before the visual search of each trial, a fixation point (black dot) was presented on the center of the laptop screen, and after $900 \mathrm{~ms}$, a stimulus was displayed. The stimulus presented for 4 seconds and participants were instructed to respond whether the target stimulus was presented and pressed the "Yes" or "No" on the keyboard as soon as possible. Participants were allowed to rest for approximately one minute between two trials. It took two-10-min to complete the feature search task and then the conjunction search task. The JACFEE was displayed for 10 seconds on the computer screen. Participants were asked to label the emotion on the multiple choices and record on the answer sheet within 20 seconds.

\section{Data reduction and statistic analysis}

When participant cannot follow the VST procedure in the practice block of 10 trials, or RTs shorter than $200 \mathrm{~ms}$ or longer than $4000 \mathrm{~ms}$, were excluded from data analyses. t-tests were used to examine the group differences in RTs and accuracy rate of the VST and accuracy rates of the JACFEE between patients with schizophrenia and normal controls. The Pearson's correlations were examined between visual search and facial expression identification by SPSS 18.0.

\section{RESULTS}

\section{Group differences in visual search performance and facial expression identification}

Regarding visual search performance, the results showed that patients with schizophrenia had longer RTs than normal controls on feature search $(\mathrm{t}=5.30, \mathrm{p}<0.001)$ and conjunction search $(\mathrm{t}=6.74, \mathrm{p}<0.001)$. Patients with schizophrenia also had lower accuracy rate than normal controls on feature se$\operatorname{arch}(\mathrm{t}=-3.32, \mathrm{p}<0.01)$ and conjunction search $(\mathrm{t}=-4.42, \mathrm{p}<$ 0.001 ). Therefore, patients with schizophrenia had significantly longer RTs and lower accuracy rate in feature search and conjunction search than normal controls (Table 2).

Regarding facial expression identification, the total accuracy rate of patients with schizophrenia was lower than that of normal controls $(t=-4.22, \mathrm{p}<0.001)$ (Figure 2). Subscales of facial expression identification showed that the accuracy rate of patients with schizophrenia were significantly lower than that of normal controls on surprised $(\mathrm{t}=-5.17, \mathrm{p}<0.001)$ and disgusted $(\mathrm{t}=-5.33, \mathrm{p}<0.001)$ (Table 2$)$.

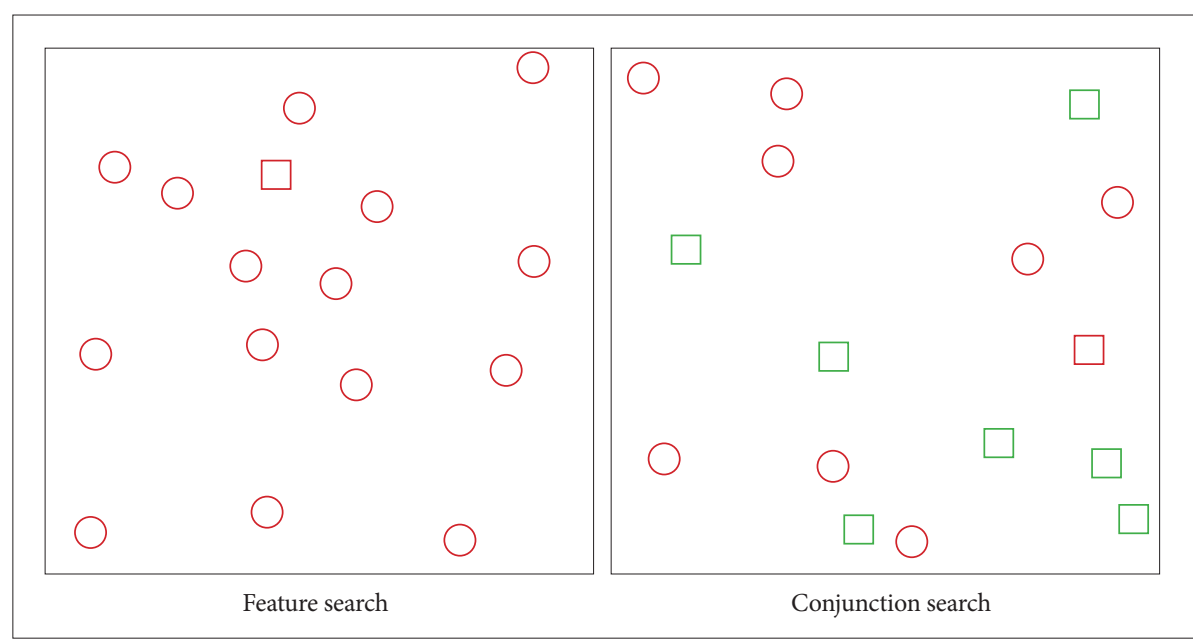

Figure 1. Example displays of feature search (left) and conjunction search (right). 
Table 2. Reaction time and accuracy rate of visual search and accuracy rate of facial expression identification in patients with schizophrenia and normal controls

\begin{tabular}{lccc}
\hline \multicolumn{1}{c}{ Mean (SD) } & Patients with schizophrenia & Normal controls \\
\hline Reaction time of visual search (ms) & & & \\
Feature search & $1732.18(503.12)$ & $870.88(353.71)$ & $1015.76(226.57)$ \\
Conjunction search & $1916.35(449.31)$ & & \\
Accuracy rate of visual search (\%) & & $96.30^{* * *}$ \\
Feature search & $64.52(33.53)$ & $96.15(12.51)$ & $-3.32^{* *}$ \\
Conjunction search & $59.45(31.01)$ & $96.37(3.86)$ & $-4.42^{* * *}$ \\
Accuracy rate of facial expression identification (\%) & $47.92(14.78)$ & $70.00(13.29)$ & $-4.22^{* * *}$ \\
Anger & $58.93(21.05)$ & $70.00(21.55)$ & -1.40 \\
Fear & $39.29(33.56)$ & $33.33(26.16)$ & 0.53 \\
Happiness & $91.07(15.83)$ & $93.33(11.44)$ & -0.44 \\
Surprised & $48.21(31.72)$ & $93.33(11.44)$ & $-5.17^{* * *}$ \\
Disgusted & $17.86(22.85)$ & $71.67(31.15)$ & $-5.33^{* * *}$ \\
Sadness & $32.14(34.57)$ & $58.33(34.93)$ & -2.03 \\
\hline
\end{tabular}

${ }^{* *} \mathrm{p}<0.01,{ }^{* * *} \mathrm{p}<0.001$

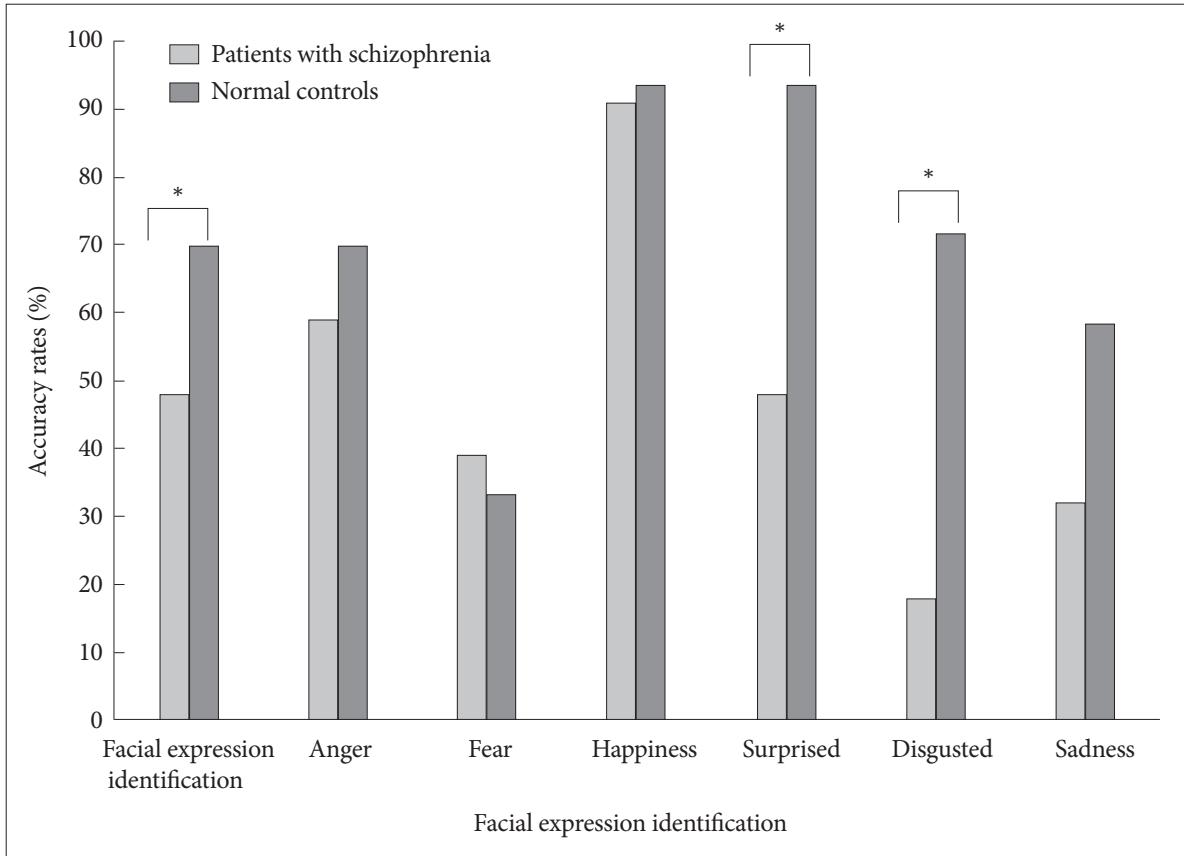

Figure 2. Accuracy rates of facial expression identification in patients with schizophrenia and normal controls.

\section{Correlations between visual search performance and facial expression identification}

For all participants, there were strong positively correlations between feature search and conjunction search on the RTs $(r=0.88, p<0.001)$ and the accuracy rate $(r=0.87, p<0.001)$. The RTs and accuracy rate of feature search and conjunction search were shown negatively correlations $(r=-0.75, p<0.001$ and $\mathrm{r}=-0.80, \mathrm{p}<0.001$ respectively) (Table 3 ).

The visual search performance was related to the facial expression identification. The RTs of feature search and conjunction search were negatively correlated with the accuracy rate of facial expression identification $(\mathrm{r}=-0.75, \mathrm{p}<0.001$ and $\mathrm{r}=$ $-0.70, p<0.001$ respectively), and the accuracy rate of feature search and conjunction search were positively correlated with the accuracy rate of facial expression identification $(r=0.60$, $\mathrm{p}<0.001$ and $\mathrm{r}=0.66, \mathrm{p}<0.001$ respectively).

For patients with schizophrenia, there were negative correlations between the accuracy rate of facial expression identifications and RTs of feature search $(r=-0.70, p<0.01)$ and conjunction search $(r=-0.67, p<0.01)$, as well as positive correlation between the accuracy rate of facial expression identifications and the accuracy rate of conjunction search $(r=0.55$, 


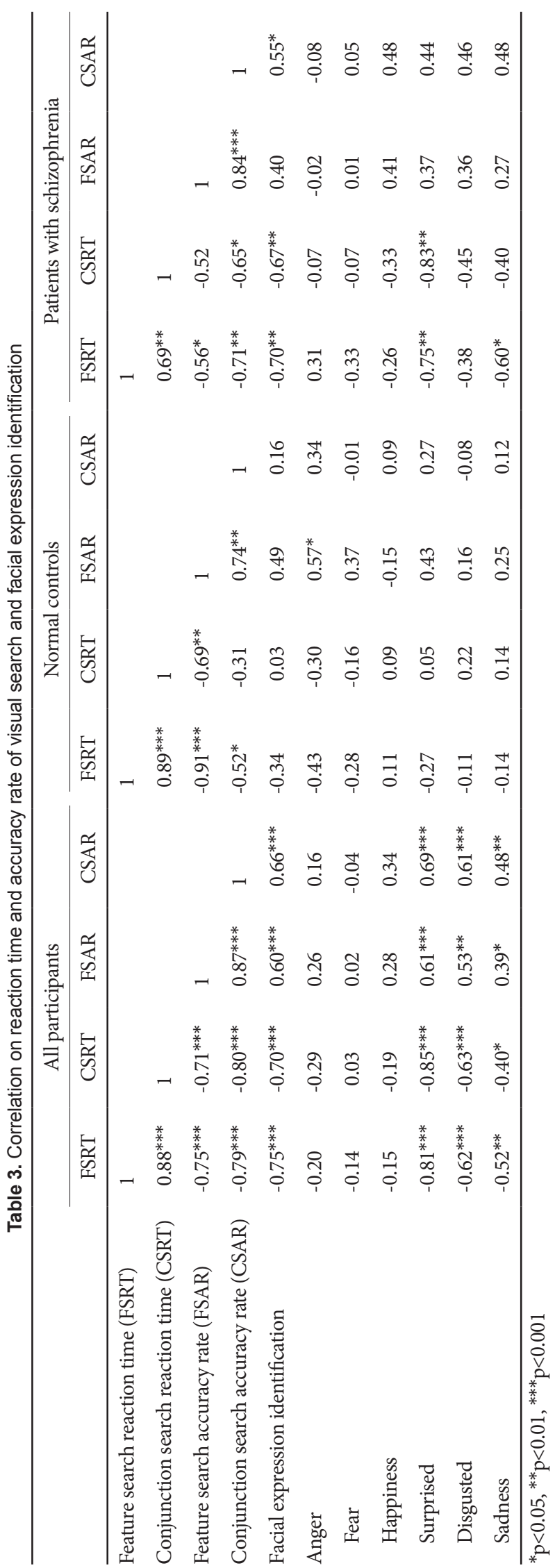

$p<0.05)$. Furthermore, regarding subscales of facial expression identification, there were negative correlations between the accuracy rate of surprised and the RTs of feature search $(r=$ $-0.75, \mathrm{p}<0.01)$ and conjunction search $(\mathrm{r}=-0.83, \mathrm{p}<0.01)$, as well as negative correlations between the accuracy rate of sadness and the RTs of feature search $(r=-0.60, p<0.05)$. However, there was no significant correlation between the VST and the JACFEE scores in normal controls.

\section{DISCUSSION}

The findings of this study showed that patients with schizophrenia had worse visual search performance both in feature search and conjunction search, as well as worse ability of facial expression identification than normal controls. In addition, there were associations between visual search performance and facial expression identification in patients with schizophrenia.

Patients with schizophrenia had deficits on visual search ability that they spent more time on visual search task but acquired a lower accuracy rate on both visual search tasks. These results were partially consistent with previous study ${ }^{3}$ that there was only a significantly difference between patients with schizophrenia and normal controls in the conjunction search rather than the feature search. However, the patients with schizophrenia in Tanaka's study were outpatients, but ours were inpatients and may have more severe psychotic symptoms that might influence the performance of visual search tasks.

The considering visual search deficit may be explained by the feature integration theory. ${ }^{9}$ According to the theory, feature search represents a preattentive process that separates the basic feature in the visual search; however, conjunction search represents a focal attention process that discriminates and integrates the visual search. Elahipanah et al. ${ }^{12}$ indicated that the perceptual grouping can help individuals to focus attention on the stimuli and then discriminate the target and distractors. Patients with schizophrenia may suffer difficulty with the perceptual grouping.

Patients with schizophrenia also had worse ability to discriminate and identify facial expression than normal controls, especially the emotion of surprised and disgusted. In patients with schizophrenia, shorter RTs of feature search and conjunction search were related to higher accuracy rates of identify the facial expression of surprised and sadness. Batty and Tay$\operatorname{lor}^{27}$ indicated the relationships between six basic facial emotional expressions and brain areas. The basal ganglia and insula was related to disgust faces; the amygdale was linked to fearful faces and sad faces; the cingulate sulcus was linked to happy faces; the orbital frontal regions was linked to anger faces. ${ }^{27}$ Wylie and Tregellas ${ }^{28}$ indicated that insula related to 
process both visual and auditory emotional information. When patients suffered the insula lesions might experience the difficulty in recognizing facial expressions, especially on disgusted, surprised, fear, anger, and sadness. ${ }^{28,29}$

In addition, there were significant relationships between visual search performance and facial expression identification only in patients with schizophrenia instead of normal controls. The deficits in visual attention or perceptual grouping may influence identify that facial expression and interrupt the interpersonal relationship. The results found that shorter RTs of visual search related to higher accuracy rate on facial expression identification, as well higher accuracy rate of visual search related to higher accuracy rate on facial expression identification. These correlations were most likely shown on surprised and sadness. Therefore, underlying information processing deficits may relate to the psychopathological mechanisms of schizophrenia, ${ }^{8}$ particularly in facial identification of surprised and sadness. Regarding the clinical implication of this study, training patients with schizophrenia concentrated on visual search task and facial expression identification may have benefits on their social function.

\section{Limitations}

Some limitations of this study should be acknowledged. First, the small sample size and the large range of the length of current hospitalization may make the results difficult to interpret and generalize to patients with schizophrenia. Second, visual attention may be one of the factors which influenced social cognition in schizophrenia, and there are still other factors such as intelligence or education. In future study, matched or case-control study design, such as controlled the psychiatry symptoms, duration of disorder, outpatients or inpatients were needed. Furthermore, exploring the pathological anatomy of schizophrenia about visual search performance and facial expression identification were needed.

\section{Acknowledgments}

We gratefully thank Shih-Kuang Chiang at National Dong Hwa University for sharing the material of Japanese and Caucasian Facial Expression of Emotion.

\section{REFERENCES}

1. Blanchard JJ, Neale JM. The neuropsychological signature of schizophrenia: generalized or differential deficit? Am J Psychiatry 1994;151: 40-48.

2. Dickinson D, Ragland JD, Gold JM, Gur RC. General and specific cognitive deficits in schizophrenia: Goliath defeats David? Biol Psychiatry 2008;64:823-827.

3. Tanaka G, Mori S, Inadomi H, Hamada Y, Ohta Y, Ozawa H. Clear distinction between preattentive and attentive process in schizophrenia by visual search performance. Psychiatry Res 2007;149:25-31.

4. Laws KR. A meta-analytic review of Wisconsin Card Sort studies in schizophrenia: general intellectual deficit in disguise? Cogn Neuropsy- chiatry 1999;4:1-30.

5. Henry JD, Crawford JR. A meta-analytic review of verbal fluency deficits in schizophrenia relative to other neurocognitive deficits. Cogn Neuropsychiatry 2005;10:1-33.

6. Dickinson D, Ramsey ME, Gold JM. Overlooking the obvious: a metaanalytic comparison of digit symbol coding task and other cognitive measures in schizophrenia. Arch Gen Psychiatry 2007;64:532-542.

7. Harris JG, Minassian A, Perry W. Stability of attention deficits in schizophrenia. Schizophr Res 2007;91:107-111.

8. Sergeant J. A Theory of Attention: An Information Processing Perspective. In: Lyon GR, Krasnegor NA, Editors. Attention, Memory, and Executive Function. Baltimore, MD: Paul H Brookes Publishing; 1996, p.57-69.

9. Treisman AM, Gelade G. A feature-integration theory of attention. Cogn Psychol 1980;12:97-136.

10. Itti L, Koch C. Computational models of visual attention. Nature Rev Neurosci 2001;2:194-203.

11. Treisman A. Features and objects in visual processing. Sci Am 1986; 255:114B-125B.

12. Elahipanah A, Christensen BK, Reingold EM. Visual selective attention among persons with schizophrenia: the distractor ratio effect. Schizophr Res 2008;105:61-67.

13. Elahipanah A, Christensen BK, Reingold EM. Attentional guidance during visual search among patients with schizophrenia. Schizophr Res 2011;131:224-230.

14. Lee TM, Cheung PP. The relationship between visual-perception and attention on Chinese with schizophrenia. Schizophr Res 2005;72:185-193.

15. Treisman A. Perceptual grouping and attention in visual-search for features and for objects. J Exp Psychol Hum Percept Perform 1982;8: 194-214.

16. Brothers L. The neural basis of primate social communication. Motiv Emot 1990;14:81-91.

17. Mandal MK, Pandey R, Prasad AB. Facial expressions of emotions and schizophrenia: a review. Schizophr Bull 1998;24:399-412.

18. Kerr SL, Neale JM. Emotion perception in schizophrenia: specific deficit or further evidence of generalized poor performance? J Abnorm Psychol 1993;102:312-318.

19. Addington J, Addington D. Facial affect recognition and information processing in Schizophrenia and bipolar disorder. Schizophr Res 1988; 32:171-181.

20. Bulter PD, Abeles IY, Weiskopf NG, Tambini A, Jalbrzikowski M, Legatt $\mathrm{ME}$, et al. Sensor contributions to impaired emotion processing in schizophrenia. Schizophr Bull 2009;35:1095-1107.

21. Kalkstein S, Hurford I, Gur RC. Neurocognition in schizophrenia. Curr Top Behav Neurosci 2010;4:373-390.

22. Cohen AS, Forbes CB, Mann MC, Blanchard JJ. Specific cognitive deficits and differential domains of social functioning impairment in schizophrenia. Schizophr Res 2006;81:227-238.

23. Penn DL, Sanna LJ, Roberts DL. Social cognition in schizophrenia: an overview. Schizophr Bull 2008;34:408-411.

24. American Psychiatric Association. Diagnostic and Statistical Manual of Mental Disorders. 4th Edition, Text Revision. Washington, DC: American Psychiatric Association; 2000.

25. Matsumoto D, Ekman P. Japanese and Caucasian Facial Expressions of Emotion (IACFEE). Unpublished Slide Set and Brochure, San Francisco State University, Department of Psychology; 1988.

26. Biehl M, Matsumoto D, Ekman P, Hearn V, Heider K, Kudoh T, et al. Matsumoto and Ekman's Japanese and Caucasian Facial Expressions of Emotion (JACFEE): reliability data and cross-national differences. J Nonverbal Behav 1997;21:3-21.

27. Batty M, Taylor MJ. Early processing of the six basic facial emotional expressions. Cogn Brain Res 2003;17:613-620.

28. Wylie KP, Tregellas JR. The role of the insula in schizophrenia. Schizophr Res 2010;123:93-104.

29. Adolphs R, Tranel D, Damasio AR. Dissociable neural systems for recognizing emotions. Brain Cogn 2003;52:61-69. 